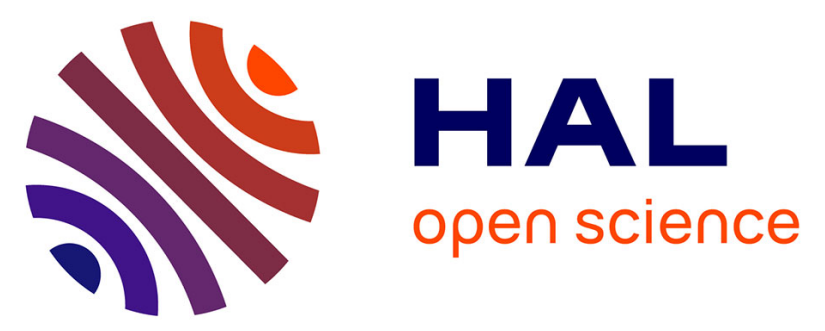

\title{
Performance Assessment of Linear Precoding for Multi-User Massive MIMO Systems on a Realistic 5G mmWave Channel
}

\author{
Mariam El Hassan, Ammar El Falou, Charlotte Langlais
}

\section{- To cite this version:}

Mariam El Hassan, Ammar El Falou, Charlotte Langlais. Performance Assessment of Linear Precoding for Multi-User Massive MIMO Systems on a Realistic 5G mmWave Channel. MENACOMM 2018 : IEEE Middle East and North Africa Communications Conference, Apr 2018, Jounieh, Lebanon. 10.1109/MENACOMM.2018.8371025 . hal-01813163

\section{HAL Id: hal-01813163 \\ https://imt-atlantique.hal.science/hal-01813163}

Submitted on 12 Jun 2018

HAL is a multi-disciplinary open access archive for the deposit and dissemination of scientific research documents, whether they are published or not. The documents may come from teaching and research institutions in France or abroad, or from public or private research centers.
L'archive ouverte pluridisciplinaire HAL, est destinée au dépôt et à la diffusion de documents scientifiques de niveau recherche, publiés ou non, émanant des établissements d'enseignement et de recherche français ou étrangers, des laboratoires publics ou privés. 


\title{
Performance Assessment of Linear Precoding for Multi-User Massive MIMO Systems on a Realistic 5G mmWave Channel
}

\author{
Mariam El Hassan*†, Ammar El Falou*, Charlotte Langlais ${ }^{\dagger}$ \\ * Faculty of Engineering, Lebanese University, Tripoli, Lebanon \\ $\dagger$ Electronics Department, IMT Atlantique, Brest, France \\ Email: mariam_elhassan@hotmail.com, ammar.elfalou@gmail.com, charlotte.langlais@imt-atlantique.fr
}

\begin{abstract}
In this paper, the performance of multi-user (MU) massive multiple-input multiple-output (MIMO) systems is evaluated in terms of bit error rate (BER). We focus on the case of a downlink single-cell scenario with linear precoding as zero forcing (ZF) and conjugate beamforming (CB). This performance evaluation is done over a statistical mmWave $5 \mathrm{G}$ propagation channel, using NYUSIM channel simulator developed by New York University in 2015. A study of the channel response characteristics is provided to define the system parameters. Simulation results in single-user scenario show better performance with an increasing number of base station antennas due to the increase of system diversity. For multi-user scenario, the performance degrade for a higher number of cell users due to inter-user interference.
\end{abstract}

Index Terms-Massive multi-user MIMO, 5G mmWave propagation, frequency selective channel, OFDM

\section{INTRODUCTION}

Mobile data transmission volume is continuously and rapidly increasing [1]. On the other hand, spectral efficiency is approaching the Shannon limit. Transmission frequency spectrum being a scarce resource, one major aim of new mobile network generations is to increase data transmission rate at the radio access network (RAN) by exploring new technologies to provide a more efficient use of the frequency spectrum.

Aiming to use mmWave bands $(>6 \mathrm{GHz})$, the $5^{\text {th }}$ mobile generation $(5 \mathrm{G})$ is going towards densification of the network and reduction of the cell size (small cell) [2], [3]. Thus, 5G systems can benefit from the large bands available in the mmWave domain. However, this move of frequency bands to $\mathrm{GHz}$ leads to new characteristics of the propagation channel in 5G compared to older generations [4].

Furthermore, multi-user (MU) massive multiple-input multiple-output (MIMO) [5] systems became of high interest as they help reaching the objectives of $5 \mathrm{G}$ rates, due to their high ability to raise network capacity and energy efficiency [6]. Therefore, it is of high interest to study MU massive MIMO systems over $5 \mathrm{G}$ mmWave propagation channels.

Many researches focused on studying MU massive MIMO chain considering a simplified Rayleigh channel (see [7] and references therein). Recent studies evaluated the performance of MU massive MIMO precoding schemes in terms of capacity and bit error rate (BER) on a more realistic mmWave 5G channel by using a geometrical model of the channel based on ray tracing [8]. However, this method for channel modeling is complex and needs lots of simplifications to make the study affordable. In our work, we consider a less complex and realistic statistical mmWave 5G propagation channel, using NYUSIM channel simulator developed by New York University [9].

The purpose of MU massive MIMO systems is to exploit the spatial dimension to ensure a beamforming of the signal in the direction of the concerned user so that each user can ideally benefit from the whole allowed bandwidth at all time. This is done by a precoding of the information at the base station (BS). Precoding aims at distributing users' data on the different antennas of the BS in order to perform beamforming of information toward the users. The BS computes the precoding matrix after estimating the channel impulse response (CIR) in a way to decrease the interfering part or to direct the useful energy in the direction of each user.

This paper aims at evaluating the performance of $\mathrm{MU}$ massive MIMO systems in terms of BER for the case of conjugate beamforming (CB) [10] and zero forcing (ZF) [11] linear precoding over a realistic $5 \mathrm{G}$ mmWave propagation using a statistical model of the channel.

Because millimeter wave propagation channels, used in this study, are frequency selective [4], we use orthogonal frequency division multiplexing (OFDM) [12] modulation to transform the frequency selective channel effect into a flat fading channel.

The rest of the paper is organized as follows. In section II, we present the system model that gives an overview of the considered transmission chain and elements, we also show the precoding schemes used in this work. Then, we describe in section III the $5 \mathrm{G}$ mmWave channel model considered in this study and we discuss the power normalization and noise power on this channel. Due to the compromise between the OFDM cyclic prefix length and the orthogonality between subcarriers, we analyze in section IV the characteristics of the power delay profile of the $5 \mathrm{G}$ propagation channel model in order to specify the cyclic prefix parameter. Having all the components of the simulated transmission chain, we show in section $\mathrm{V}$ the simulation results and give an analysis of these results. Section VI concludes this work. 


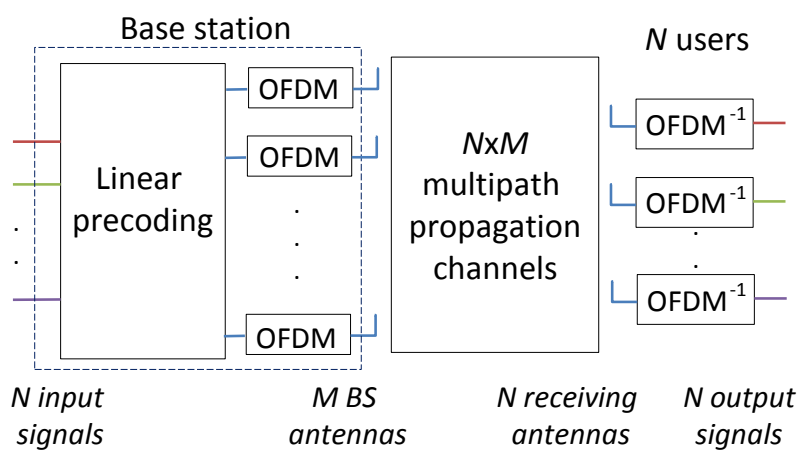

Fig. 1. System model.

\section{System Model}

We consider a MU massive MIMO system over OFDM modulation for a single cell downlink propagation where the BS has $M$ transmitting antennas serving simultaneously the $N$ single-antenna users of the cell as depicted in Fig. 1. We consider OFDM modulation over $P$ subcarriers and transmission over the $5 \mathrm{G}$ propagation channel model that we describe in section III, operating at a frequency of $28 \mathrm{GHz}$ and a bandwidth of $800 \mathrm{MHz}$.

Due to OFDM modulation, each of the $M \times N$ frequency selective channels is ideally equivalent to a flat fading channel over each subcarrier $p$ if the cyclic prefix is larger than the channel length [12]. Thus, precoding can be done independently on each of these subcarriers.

Let $\mathbf{H}_{p} \in \mathbb{C}^{N \times M}$ be the matrix containing the coefficients of the $M \times N$ propagation channel at the subcarrier $p$ and $\mathbf{W}_{p} \in \mathbb{C}^{M \times N}$ the precoding matrix that distributes the $N$ symbols sent to the users on the $M$ transmitting antennas at subcarrier $p$. Let $\mathbf{s}_{p} \in \mathbb{C}^{N}$ be the vector containing the $N$ symbols sent to the $N$ users over the subcarrier $p$ and $\mathbf{r}_{p} \in \mathbb{C}^{N}$ the vector containing the received signals by the $N$ users over the subcarrier $p$. We can model the system by:

$$
\mathbf{r}_{p}=\sqrt{\eta}_{p} \mathbf{H}_{p} \mathbf{W}_{p} \mathbf{s}_{p}+\mathbf{n}_{p}
$$

where $\mathbf{n}_{p} \in \mathbb{C}^{N}$ is the vector of additive white Gaussian noise (AWGN) noise that affects the symbols of $N$ users at subcarrier $p$ and $\eta_{p}$ is the normalization factor of the precoding matrix, equal to $\left.1 / \mathrm{E}\left[\operatorname{Tr}\left(\mathbf{W}_{p}^{H} \mathbf{W}_{p}\right)\right]\right]$ (E[.] stands for the mathematical expectation, $\operatorname{Tr}($.$) for the trace of the matrix$ and the superscript ${ }^{H}$ for the conjugate transpose).

The received signal at each user is thus composed of a useful part that contains the symbol sent to this user and an interfering part that is composed of the $N-1$ symbols sent to the other users of the cell. The BS computes the precoding matrix after estimating the CIR in a way to decrease the interfering part or to direct the useful energy in the direction of each user. In our work, we assume that the CIR is completely known at the BS and at the receivers. Achieving a perfect channel estimation at the BS is conventionally obtained in the TDD (Time Division Duplexing) mode thanks to channel reciprocity [13].
In this study we consider two types of precoders: Conjugate Beamforming (CB) and Zero Forcing (ZF).

Conjugate Beamforming Precoding [10]: Also known as Matched Filtering precoding (MF). The precoding matrix is expressed by:

$$
\mathbf{W}_{p}^{\mathrm{MF}}=\mathbf{H}_{p}^{H}
$$

So, the expression of $\mathbf{r}_{p}$ in this case is:

$$
\mathbf{r}_{p}=\sqrt{\eta}_{p} \mathbf{H}_{p} \mathbf{H}_{p}^{H} \mathbf{s}_{p}+\mathbf{n}_{p}
$$

Conjugate Beamforming permits to increase the signal to interference ratio (SIR) with the increase of the number of transmitting antennas. Its objective is to maximise the power of the received signal at each user.

Zero Forcing Precoding [11]: The precoding matrix is expressed as:

$$
\mathbf{W}_{p}^{\mathrm{ZF}}=\mathbf{H}_{p}^{H}\left(\mathbf{H}_{p} \mathbf{H}_{p}^{H}\right)^{-1}
$$

If $\mathbf{H}_{p} \mathbf{H}_{p}^{H}$ is an invertible matrix, the zero forcing precoding makes the interfering part equal to zero, at the price of a complex computation of $\mathbf{W}_{p}$ and noise enhancement.

\section{Propagation Channel Model}

\section{A. NYUSIM simulator}

We consider simulations on a realistic $5 \mathrm{G}$ mmWave propagation channel using NYUSIM simulator developed by New York University [9]. The simulator provides a statistical channel model suitable for Monte Carlo simulations. The simulator model is based on a large number of measurements from the year 2011 to 2015 in New York City at millimeter frequency bands in urban environments and can therefore give insights on real performance. The simulator models the channel on an $800 \mathrm{MHz}$ bandwidth on different mmWave frequencies. In our work, a frequency of $28 \mathrm{GHz}$ is considered. A full description of the measurements and the statistical model of the simulator in single-input single-output (SISO) systems is provided in [14]. The simulator also gives a model of MIMO propagation channels from the SISO model as described in [15].

\section{B. MIMO channels correlation and power distribution}

As we are working with MIMO systems, we have $M \times N$ modeled propagation channels. Note that for MU MIMO systems, we generate $N$ independent $M \times 1$ multiple-input singleoutput (MISO) channels from the MIMO channel model, since users channels are not correlated due to large distances between users with respect to the propagation wavelength (approximately $10 \mathrm{~mm}$ in our case) . In order to model a MISO channel, the simulator generates one SISO CIR of several multipath components. The $M-1$ other links are generated from the information on the antenna array geometry. So, the $M$ channels of each MISO channel are correlated and have the same number of multipath components while each user can receive data over different MISO channel length.

For the transmitted power, we note that it is conserved in MU MIMO since it is distributed on the transmitting antennas. As we are dealing with BER simulations, where we consider the noise power effect, we omit the effect of path loss on the 

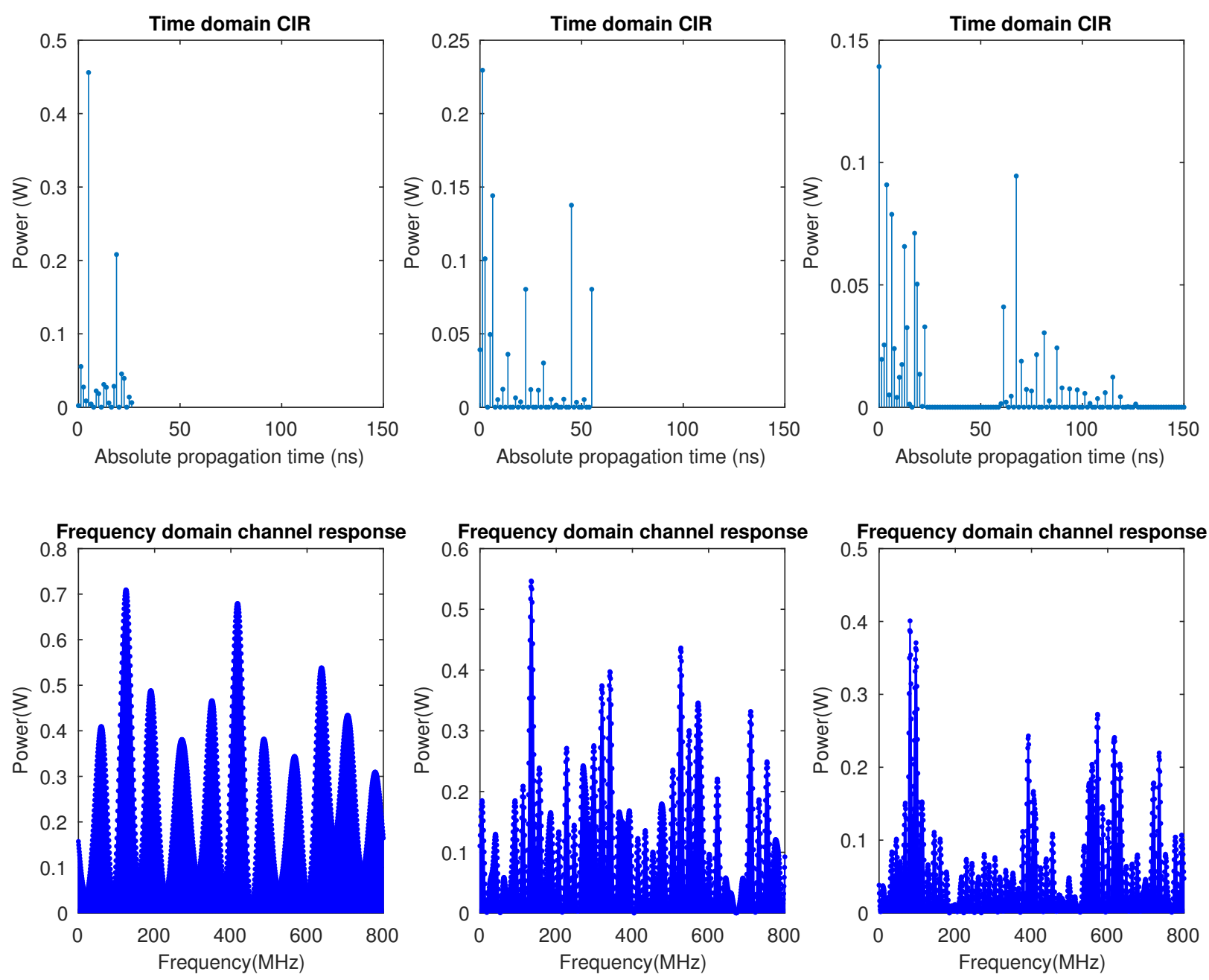

Fig. 2. Example of three CIR tests from the SISO CIR model in time and frequency domain.

transmitted signal so that the transmitted power is conserved at each receiver.

\section{Statistical Propagation ChanNel ANALYSis AND PARAMETER DEFINITIONS}

The propagation channel model considers a random number of multipath components where the delay between each multipath component follows an exponential distribution, yielding to very long delay spreads. However, the model also considers that the power distribution of these components is exponentially decreasing with the increase of the delay. So, the probability of having high power multipath components with long delay spread is very low. Indeed, in Fig. 2, we show three independent realizations of the CIR in the time and frequency domains.

If we want to have ideal OFDM transmission equivalent to a transmission on a flat fading channel as in (1), we have to take into consideration the maximum delay spread. This poses high constraints on the number of OFDM subcarriers and the cyclic prefix length. A long cyclic prefix ensures interferencefree transmission but at the cost of a loss in spectral efficiency.

For this purpose, we study in this section the statistics of the SISO power delay profiles in order to define the cyclic prefix length, knowing that MIMO channels have equivalent power distributions.

We show in Fig. 3 the statistics of the maximum power and the average total power of the multipath components after a certain delay, defined as the threshold in $x$ axis, averaged over 1000 channel tests for each threshold independently. As expected from the statistical channel model, we remark that the power of the multipath components is decreasing rapidly with the increasing of the delay. Thus, when the delay threshold is higher than $250 \mathrm{~ns}$, the remaining average total power is less than $-23 \mathrm{~dB}$, which constitutes less than $0.005 \%$ of the total received power. For the rest of the paper, we consider a cyclic 

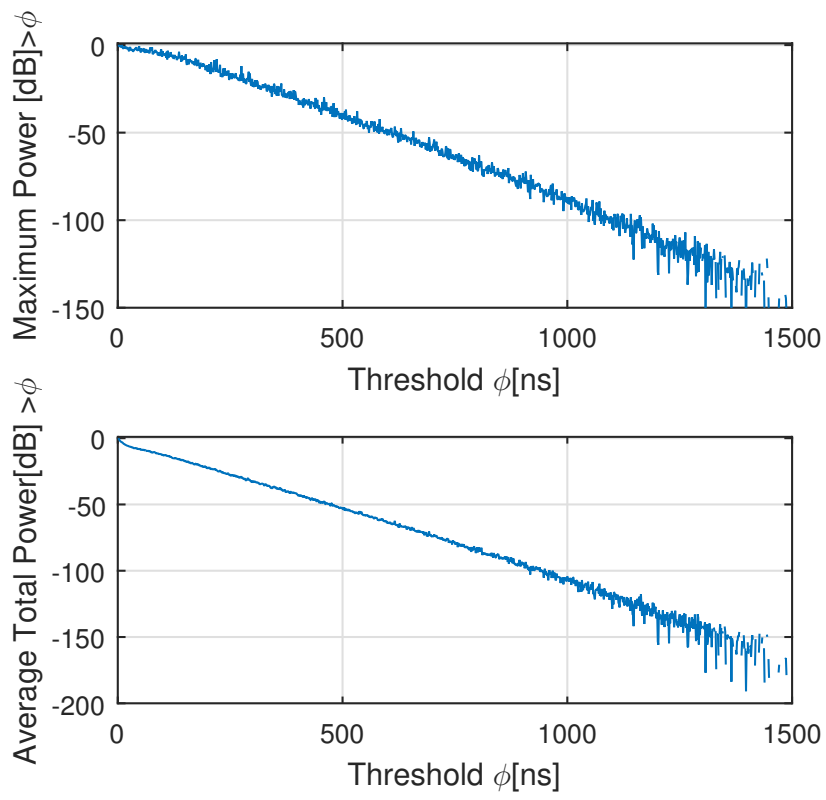

Fig. 3. Statistics of the maximum and the average total power.

prefix length of $250 \mathrm{~ns}$ assuming that the power of multipath components after $250 \mathrm{~ns}$ is negligible. This will be verified by Monte-Carlo simulations at the next section.

\section{Simulation Results}

In this section, we present the simulation results for the downlink single cell MU massive MIMO-OFDM system described in section II. Results are evaluated on the mmWave 5G channel described in section III for the case of ZF and CB precoding with a cyclic prefix of 250 ns with $P=1024$ subcarriers and 4QAM modulation. The channel is time sampled at $1.25 \mathrm{~ns}$ as the system bandwidth is $800 \mathrm{MHz}$. The cyclic prefix length is thus equivalent to 200 time samples. We consider that the channel is constant during one OFDM symbol, so that we launch a new realization from the channel model for each OFDM symbol. A block consists of 100 independent OFDM symbols that can potentially interfere with each other depending on the length of the cyclic prefix. We consider a perfect knowledge of the CSI at the BS and at the receivers. Study of imperfect channel estimation is left for future works.

\section{A. Single-user scenario}

In this section, we consider the single-user scenario i.e., the scenario where only one user $(N=1)$ is served by the BS having $M$ transmitting antennas. In Fig. 4 and 5, we plot the BER performance respectively in the case of ZF and $\mathrm{CB}$ linear precoding. As for a reference, we also plot the BER of the 4QAM for an AWGN channel. With only $M=1$ antenna, the BER performance is poor since the system is not able to exploit neither the frequency diversity (no channel coding is included), nor the spatial diversity.

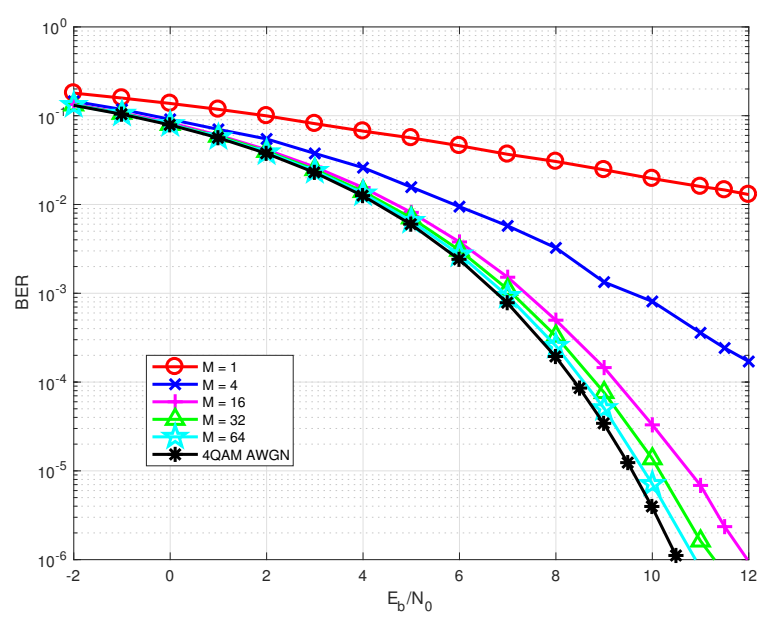

Fig. 4. BER performance for single-user scenario with ZF precoding.

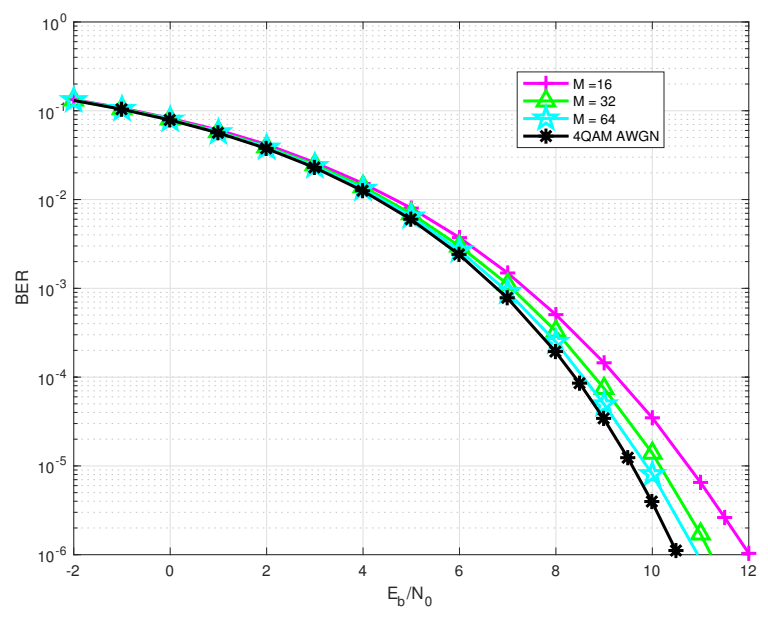

Fig. 5. BER performance for single-user scenario with $\mathrm{CB}$ precoding.

Increasing the number of transmitting antennas $M$ increases the spatial diversity at the receiver and thus improves the BER performance. Finally with $M=64$ antennas, the BER performance over the mmWave $5 \mathrm{G}$ channel is close to the one obtained over the AWGN channel, which verifies that the system is correctly designed, especially from the cyclic prefix point of view. In this multiuser interference free configuration, the two precoders obviously perform in the same way.

\section{B. Multi-user scenario}

In Fig. 6 and 7, we study the effect of increasing the number of cell users $(N \geq 1)$ on BER performance for a massive number of transmitting antennas e.g., $M=64$. We remark a degradation of BER performance when the number of users increase. In fact, when the number of users increases, the interuser interference effect is more important and thus degrades the system performance. However, for a number of users that is much less than the number of antennas, the performance is approaching the AWGN channel case. 


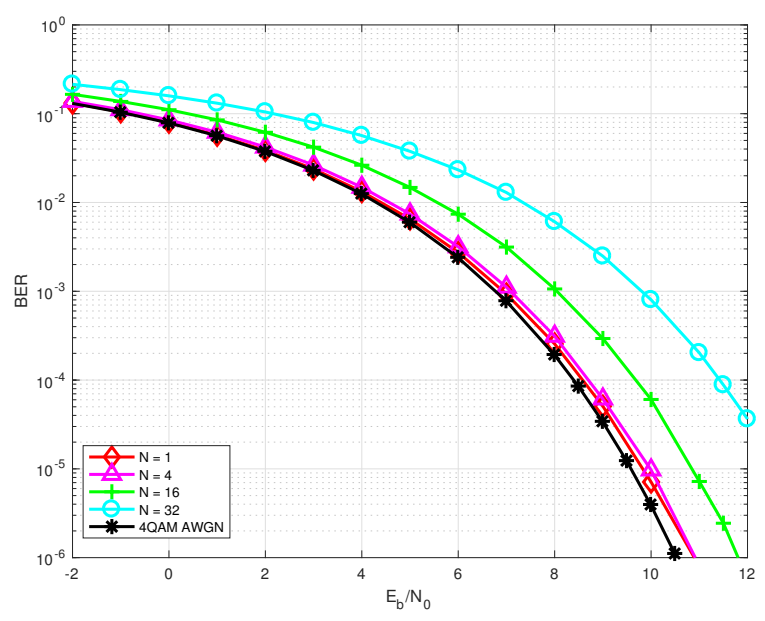

Fig. 6. BER performance for MU massive MIMO systems with $M=64$ transmitting antennas using ZF precoding.

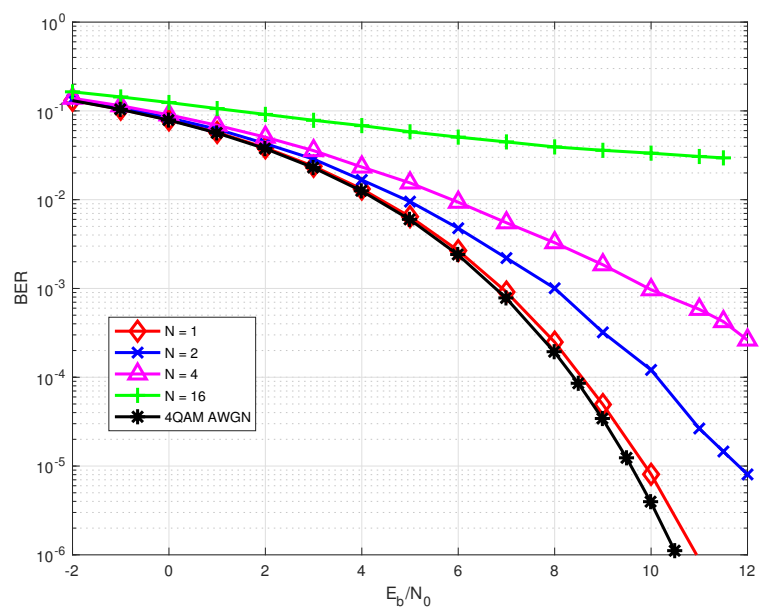

Fig. 7. BER performance for MU massive MIMO systems with $M=64$ transmitting antennas using $\mathrm{CB}$ precoding.

For the $\mathrm{CB}$ case the multi-user interference increases with the increase of the number of cell users. In fact, for the $\mathrm{CB}$ case, the interfering part tends to zero when the ratio $M / N$ is larger. For the $\mathrm{ZF}$ precoding, however, the multiuser interference is always zero but if the ratio $M / N$ is not high an amount power is lost.

\section{CONCLUSION}

In this paper, we studied the performance in terms of BER of a single-cell downlink MU massive MIMO-OFDM system over a $5 \mathrm{G}$ mmWave propagation channel. This propagation channel is generated by a statistical channel simulator NYUSIM suitable for Monte Carlo simulations. We first studied the statistics of the power delay profile of this channel in order to specify the number of significant paths in order to optimize the cyclic prefix parameter. We thus specified a cyclic prefix of 200 time samples. Simulation results for single-user scenario show the impact of increasing the number of base station antennas that enhance the system performance due to the higher diversity. For multi-user scenario, a degradation of performance is obtained with the increase of the number of cell users. This degradation is more significant with $\mathrm{CB}$ precoding than with $\mathrm{ZF}$ precoding.

\section{REFERENCES}

[1] Cisco Visual Networking Index: Global Mobile Data Traffic Forecast Update, 2016-2021 White Paper, Cisco. [Online]. Available: https://www.cisco.com/c/en/us/solutions/collateral/serviceprovider/visual-networking-index-vni/mobile-white-paper-c11520862.html. [Accessed: 19-Sep-2017].

[2] N. Bhushan, J. Li, D. Malladi, R. Gilmore, D. Brenner, A. Damnjanovic, R. Sukhavasi, C. Patel and S. Geirhofer, "Network densification: the dominant theme for wireless evolution into 5G", IEEE Communications Magazine, vol. 52, no. 2, pp. 82-89, Feb. 2014.

[3] T. Nakamura, S. Nagata, A. Benjebbour, Y. Kishiyama, T. Hai, S. Xiaodong, Y. Ning and L. Nan, "Trends in small cell enhancements in LTE advanced", IEEE Communications Magazine, vol. 51, no. 2, pp. 98-105, Feb. 2013.

[4] R. C. Daniels and R. W. H. Jr, " $60 \mathrm{GHz}$ wireless communications: emerging requirements and design recommendations", IEEE Vehicular Technology Magazine, vol. 2, no. 3, pp. 41-50, Sep. 2007.

[5] E. G. Larsson, O. Edfors, F. Tufvesson, and T. L. Marzetta, "Massive MIMO for next generation wireless systems", IEEE Communications Magazine, vol. 52, no. 2, pp. 186-195, Feb. 2014.

[6] J.G. Andrews, S. Buzzi, W. Choi, S.V. Hanly, A. Lozano, A.C. Soong and J.C. Zhang, "What Will 5G Be?", IEEE Journal on Selected Areas in Communications, vol. 32, no. 6, pp. 1065-1082, Jun. 2014

[7] D. L. Colon, F. H. Gregorio, and J. Cousseau, "Linear precoding in multi-user massive MIMO systems with imperfect channel state information", in Proc. Workshop on Information Processing and Control (RPIC), 2015.

[8] A. Roze, M. Helard, M. Crussiere, and C. Langlais, "Linear Precoder Performance for Massive MIMO Systems in near LOS Environments: Application to mmWave Transmission", in Proc. European Wireless 2015; 21th European Wireless Conference, 2015.

[9] NYUSIM Download, NYU WIRELESS, version 1.4, march 2017.[available online] wireless.engineering.nyu.edu $/ 5 \mathrm{~g}$-millimeter-wave-channelmodeling- software/ viewed 3 march 2017.

[10] M. Joham, W. Utschick, and J. A. Nossek, "Linear transmit processing in MIMO communications systems", IEEE Transactions on Signal Processing, vol. 53, no. 8, pp. 2700-2712, Aug. 2005.

[11] A. Wiesel, Y. C. Eldar, and S. Shamai, "Zero-Forcing Precoding and Generalized Inverses", IEEE Transactions on Signal Processing, vol. 56, no. 9, pp. 4409-4418, Sep. 2008.

[12] R.V. Nee, and R. Prasad. "OFDM for wireless multimedia communications", Artech House, Inc., 2000.

[13] B. Vojcic and W. M. Jang, "Transmitter precoding in synchronous multiuser communications", IEEE Trans. Commun., vol. 46, no. 10, pp. 1346-1355, Oct. 1998.

[14] M. K. Samimi and T. S. Rappaport, "3-D Millimeter-Wave Statistical Channel Model for 5G Wireless System Design", IEEE Transactions on Microwave Theory and Techniques, vol. 64, no. 7, pp. 2207-2225, Jul. 2016.

[15] M. K. Samimi, S. Sun, and T. S. Rappaport, "MIMO channel modeling and capacity analysis for 5G millimeter-wave wireless systems", in Proc. 10th European Conference on Antennas and Propagation (EuCAP), 2016, pp. 1-5. 